\title{
DAMAGE MODELING USING STRAIN GRADIENT BASED FINITE ELEMENT FORMULATION
}

\author{
Filip Putar, Jurica Sorić, Tomislav Lesičar and Zdenko Tonković \\ Faculty of Mechanical Engineering and Naval Architecture, University of Zagreb \\ Ivana Lučića 5, 10002 Zagreb, Croatia \\ e-mail: \{filip.putar, jurica.soric, tomislav.lesicar, zdenko.tonkovic\}@fsb.hr
}

Keywords: Damage, Softening, $C^{1}$ Continuity Finite Element, Strain Gradient Theory, Heterogeneous Material.

\begin{abstract}
The present study deals with the modeling of quasi-brittle softening phenomena using the two dimensional $C^{l}$ continuity triangular finite element. The formulation of damage model is based on an isotropic damage law applied to the higher-order stress-strain constitutive relations originating from the full strain gradient theory. Both homogeneous and heterogeneous materials are considered by employing the second-order homogenization procedure to obtain the required constitutive matrices. For this purpose, two different heterogeneous representative volume elements are employed, where the results of the softening analyses are compared with those obtained for the corresponding homogeneous materials with equivalent internal length scales. The derived finite element formulation is implemented into the finite element program ABAQUS using user subroutines. Finally, accuracy and efficiency of the proposed higher-order gradient model are demonstrated by the modeling of a stretched plate weakened in the middle, usually used as a benchmark in strain softening analyses.
\end{abstract}




\section{INTRODUCTION}

A large number of engineering materials, such as high-strength steels, polymers, composites, concrete and rocks can be classified as quasi-brittle, since they exhibit negligible plastic behavior before and after the damage is initiated, causing the material to soften immediately after reaching the maximum elastic deformation. It is well-known that the application of the classical continuum mechanics cannot properly resolve strain softening because it leads to the local loss of positive definiteness of the material tangent stiffness. As a consequence, the differential equations which describe the deformation process may result in the loss of ellipticity. Mathematical description of the model than becomes ill-posed and numerical solutions do not converge to a physically meaningful solution [1]. In view of the finite elements, solutions are completely dependent on the discretization, with respect to mesh refinement and mesh alignment. Energy dissipated in the fracture process tends to zero when the size of the elements involved in the softening process is reduced, and the localization zone exhibits an extreme tendency to propagate along the mesh lines [2]. Various regularization techniques have been developed in the past few decades to overcome this problem. Most of them are based on the improvement of the classical continuum model, precisely on its enrichment with the internal length scale parameters in several different ways. Micropolar [3] and viscoplastic theory [4] can preserve the ellipticity only in some specific cases, while the theories related to the nonlocal material behavior have been shown to be the most versatile. In the case of the nonlocal models, stress at a material point does not depend only on the strain and other state variables at this point, as it is the case with the classical continuum theory, but also on the strains and other state variables of the points in the surrounding area. The magnitude of this interaction is described by the aforementioned internal length scale parameter, which represents the microstructure of the material [5]. Basically, there are two different approaches when it comes to describing of non-locality in the model, integral and gradient-enhanced approach. The integral approach, introduced in [6], is based on spatial averaging of the state variables, typically strains, in the finite neighborhood of a certain point, leading to the very complicated constitutive relations made of convolution-type integrals. The gradient approach enhances the constitutive relation either by incorporation of the straingradients or by introduction of both strain-gradients and their stress conjugates. In case when only strain-gradients are used as an enhancement of the constitutive relation, explicit and implicit gradient formulations are usually used when dealing with softening, either in elasticity context [7], plasticity context [8] or in the analysis of the elastic wave propagation [9]. The second type of gradient approaches where both strain-gradients and their stress conjugates enter the constitutive relation has been employed less often, mainly because it is numerically more complex. In the recent developments, higher-order stress-strain theory has been employed in the context of a damage modeling of an infinitely long bar, where the authors concluded that the addition of the higher-order stress terms results in stabilizing the positive definiteness of tangent stiffness moduli when entering the strain softening regime. In such a way physically consistent solutions can be ensured and strain-softening phenomenon can be realistically reproduced [10]. Further development from one-dimensional to multidimensional simulation of a localized failure process has been made in [11]. In [10] and [11] element-free Galerkin (EFG) meshless method has been used for finding the approximate solutions to the corresponding boundary value problems. Another advantage of the higherorder stress-strain theory is that it can easily introduce material heterogeneity in the constitutive relations through the non-diagonal higher order material stiffness tangents [12]. The stiffness tangents can be obtained by applying the second-order homogenization technique on the representative volume element (RVE) [13]. 
This paper presents formulation and numerical implementation of the higher-order stressstrain damage theory into the $C^{1}$ continuity displacement based finite element developed in [14]. Herein, continuum damage mechanics is realized in its simplest form through isotropic damage model. Similar consideration of quasi-brittle damage response has been presented in [15]. The proposed damage algorithm is verified on the benchmark example where both homogeneous and heterogeneous materials are employed.

\section{FORMULATION OF THE $C^{\mathbf{1}}$ FINITE ELEMENT FOR SOFTENING ANALYSIS}

In this section, an extension of the $C^{1}$ continuity finite element presented in the authors' former work [14] for the application in a softening regime is described. The developed finite element formulation is based on a small strain second gradient continuum theory for which more details can be found in [16]. Here the basic relations of the damage constitutive model are presented, and afterwards derivation of the finite element with a softening behavior is shown.

\subsection{Damage constitutive model}

The isotropic damage constitutive model describing the reduction of the elastic stiffness properties in quasi-brittle materials is based on the following stress-strain relationship [17]

$$
\boldsymbol{\sigma}=(1-D) \mathbf{C} \boldsymbol{\varepsilon}
$$

where $D$ is a scalar damage variable ranging from 0 (undamaged) to 1 (damaged), while $\boldsymbol{\sigma}, \boldsymbol{\varepsilon}$ and $\mathbf{C}$ are tensors referring to Cauchy stress, strain and elastic stiffness, respectively. In this contribution, a simple linear damage evolution law is chosen [5]

$$
D=\left\{\begin{array}{lc}
\frac{k_{u}\left(\varepsilon_{e q v}-k_{0}\right)}{\varepsilon_{e q v}\left(k_{u}-k_{0}\right)} & k_{0} \leq \varepsilon_{e q v} \leq k_{u}, \\
1 & \varepsilon_{e q v}>k_{u}
\end{array}\right.
$$

where $k_{0}$ and $k_{u}$ are the material parameters representing the threshold strain at which damage is initiated, and the strain at which material completely loses its stiffness, respectively. $\varepsilon_{\text {eqv }}$ is the equivalent elastic strain measure which, considering the damage only due to tensile strains, can be expressed as

$$
\varepsilon_{e q v}=\sqrt{\left(\varepsilon_{1}\right)^{2}+\left(\varepsilon_{2}\right)^{2}} \text { for } \varepsilon_{1}, \varepsilon_{2}>0
$$

with $\varepsilon_{1}$ and $\varepsilon_{2}$ as principal strain components of the strain tensor $\boldsymbol{\varepsilon}$.

\section{2 $C^{1}$ triangular finite element for softening analysis}

The $C^{1}$ continuity plane strain triangular finite element proposed in [14] shown in Fig. 1 is used in the formulation derived in this contribution. The element has three nodes and 36 degrees of freedom with the displacement field approximated by the full fifth order polynomial. The nodal degrees of freedom are the two displacements and their first- and second-order derivatives with respect to the Cartesian coordinates. 


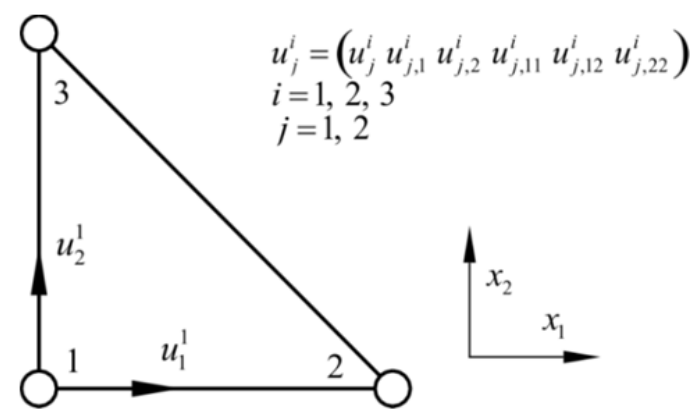

Figure 1: $C^{1}$ triangular finite element [14]

As usual, the element equations are derived from the principle of virtual work, which may be expressed for the strain gradient continuum as

$$
\int_{A} \delta \boldsymbol{\varepsilon}^{T} \boldsymbol{\sigma} \mathrm{d} A+\int_{A} \delta \boldsymbol{\eta}^{T} \boldsymbol{\mu} \mathrm{d} A=\int_{s} \delta \mathbf{u}^{T} \mathbf{t} \mathrm{d} s+\int_{s} \delta\left(\operatorname{grad} \mathbf{u}^{T}\right) \mathbf{T} \mathrm{d} s,
$$

where $A$ and $s$ are area and perimeter of the element, respectively. In Eq. (4), $\boldsymbol{\eta}$ represents the second-order strain tensor containing second derivatives of the displacement vector $\mathbf{u}$, while $\boldsymbol{\mu}$ is the work conjugate of the second-order strain, the so-called second-order stress or double stress tensor. $\mathbf{t}$ and $\mathbf{T}$ are the traction tensor and the double traction tensor, respectively. The stress and the second-order stress increments, $\Delta \boldsymbol{\sigma}$ and $\Delta \boldsymbol{\mu}$, are computed by the incremental constitutive relations which, for the undamaged material are defined as

$$
\begin{aligned}
& \Delta \boldsymbol{\sigma}=\mathbf{C}_{\sigma \varepsilon} \Delta \boldsymbol{\varepsilon}+\mathbf{C}_{\sigma \eta} \Delta \boldsymbol{\eta}, \\
& \Delta \boldsymbol{\mu}=\mathbf{C}_{\mu \varepsilon} \Delta \boldsymbol{\varepsilon}+\mathbf{C}_{\mu \eta} \Delta \boldsymbol{\eta} .
\end{aligned}
$$

Herein, $\mathbf{C}_{\sigma \varepsilon}, \mathbf{C}_{\sigma \eta}, \mathbf{C}_{\mu \varepsilon}$ and $\mathbf{C}_{\mu \eta}$ are the constitutive tangent matrices which can be computed from the appropriate RVE using the second-order homogenization procedure. In case of material homogeneity, the constitutive tangent matrices $\mathbf{C}_{\sigma \eta}$ and $\mathbf{C}_{\mu \varepsilon}$ are assumed to be zero [12]. The remaining two tangent stiffness matrices can be found analytically [18], which may be written symbolically in the form

$$
\begin{aligned}
& \mathbf{C}_{\sigma \varepsilon}=\mathbf{C}_{\sigma \varepsilon}(E, v), \\
& \mathbf{C}_{\mu \eta}=\mathbf{C}_{\mu \eta}(E, v, L),
\end{aligned}
$$

where $L$ denotes the size of the microstructural representative volume element. It is wellknown that, in the second-order computational homogenization scheme, the RVE size $L$ is linked to the internal length scale $l$ of the higher-order continuum as

$$
l^{2}=\frac{L^{2}}{12} .
$$

After application of the isotropic damage law, Eq. (1), to the constitutive relations, Eq. (5), the constitutive damage model may be expressed in the form

$$
\begin{aligned}
& \Delta \boldsymbol{\sigma}=\left(1-D^{i-1}\right)\left(\mathbf{C}_{\sigma \varepsilon} \Delta \boldsymbol{\varepsilon}+\mathbf{C}_{\sigma \eta} \Delta \boldsymbol{\eta}\right)-\Delta D\left(\mathbf{C}_{\sigma \varepsilon} \boldsymbol{\varepsilon}^{i-1}+\mathbf{C}_{\sigma \eta} \boldsymbol{\eta}^{i-1}\right), \\
& \Delta \boldsymbol{\mu}=\left(1-D^{i-1}\right)\left(\mathbf{C}_{\mu \varepsilon} \Delta \boldsymbol{\varepsilon}+\mathbf{C}_{\mu \eta} \Delta \boldsymbol{\eta}\right)-\Delta D\left(\mathbf{C}_{\mu \varepsilon} \boldsymbol{\varepsilon}^{i-1}+\mathbf{C}_{\mu \eta} \boldsymbol{\eta}^{i-1}\right),
\end{aligned}
$$


where the stress and the second-order stress increments are computed from the values of the last converged equilibrium state $(i-1)$. Here the incremental change of the damage variable is approximated by

$$
\Delta D=\left(\frac{\mathrm{d} D}{\mathrm{~d} \boldsymbol{\varepsilon}}\right)^{i-1} \Delta \boldsymbol{\varepsilon} .
$$

Employing the relations given in [14], the strain and second-order strain increments can be expressed in terms of the nodal displacement increment vector $\Delta \mathbf{v}$ by the relations

$$
\begin{aligned}
\Delta \boldsymbol{\varepsilon} & =\mathbf{B}_{\varepsilon} \Delta \mathbf{v}, \\
\Delta \boldsymbol{\eta} & =\mathbf{B}_{\eta} \Delta \mathbf{v},
\end{aligned}
$$

where $\mathbf{B}_{\varepsilon}$ and $\mathbf{B}_{\eta}$ are the matrices containing corresponding the first and second derivatives of the element interpolation functions. Substituting Eqs. (8)-(10) into Eq. (4), and after some straightforward manipulation, the following finite element equation is obtained

$$
\left(\mathbf{K}_{\varepsilon \varepsilon}+\mathbf{K}_{\varepsilon \eta}+\mathbf{K}_{\eta \varepsilon}+\mathbf{K}_{\eta \eta}\right) \Delta \mathbf{v}=\mathbf{F}_{\mathrm{e}}-\mathbf{F}_{\mathrm{i}}
$$

where the particular element stiffness matrices are defined as

$$
\begin{aligned}
\mathbf{K}_{\varepsilon \varepsilon} & =\int_{A} \mathbf{B}_{\varepsilon}^{T}\left[\left(1-D^{i-1}\right) \mathbf{C}_{\sigma \varepsilon}-\mathbf{C}_{\sigma \varepsilon} \boldsymbol{\varepsilon}^{i-1}\left(\frac{\mathrm{d} D}{\mathrm{~d} \boldsymbol{\varepsilon}}\right)^{i-1}-\mathbf{C}_{\sigma \eta} \eta^{i-1}\left(\frac{\mathrm{d} D}{\mathrm{~d} \boldsymbol{\varepsilon}}\right)^{i-1}\right] \mathbf{B}_{\varepsilon} \mathrm{d} A, \\
\mathbf{K}_{\varepsilon \eta} & =\int_{A} \mathbf{B}_{\varepsilon}^{T}\left(1-D^{i-1}\right) \mathbf{C}_{\sigma \eta} \mathbf{B}_{\eta} \mathrm{d} A, \\
\mathbf{K}_{\eta \varepsilon} & =\int_{A} \mathbf{B}_{\eta}^{T}\left[\left(1-D^{i-1}\right) \mathbf{C}_{\mu \varepsilon}-\mathbf{C}_{\mu \varepsilon} \boldsymbol{\varepsilon}^{i-1}\left(\frac{\mathrm{d} D}{\mathrm{~d} \boldsymbol{\varepsilon}}\right)^{i-1}-\mathbf{C}_{\mu \eta} \eta^{i-1}\left(\frac{\mathrm{d} D}{\mathrm{~d} \boldsymbol{\varepsilon}}\right)^{i-1}\right] \mathbf{B}_{\varepsilon} \mathrm{d} A, \\
\mathbf{K}_{\eta \eta} & =\int_{A} \mathbf{B}_{\eta}^{T}\left(1-D^{i-1}\right) \mathbf{C}_{\mu \eta} \mathbf{B}_{\eta} \mathrm{d} A .
\end{aligned}
$$

The external and internal nodal force vectors, $\mathbf{F}_{\mathrm{e}}$ and $\mathbf{F}_{\mathrm{i}}$, in Eq. (11) are defined as

$$
\begin{aligned}
& \mathbf{F}_{\mathrm{e}}=\int_{s}\left(\mathbf{N}^{T} \mathbf{t}+\operatorname{grad} \mathbf{N}^{T} \mathbf{T}\right) \mathrm{d} s, \\
& \mathbf{F}_{\mathrm{i}}=\int_{A}\left(\mathbf{B}_{\varepsilon}^{T} \boldsymbol{\sigma}^{i-1}+\mathbf{B}_{\eta}^{T} \boldsymbol{\mu}^{i-1}\right) \mathrm{d} A .
\end{aligned}
$$

\subsection{Analysis procedure}

The block diagram which concisely shows the analysis procedure is presented in Fig. 2. The first step of the analysis is a preprocessing step and includes application of the secondorder homogenization procedure to the appropriate RVE in order to obtain required constitutive stiffness tensors. As the linear elastic material behavior is considered in the presented damage analysis, the homogenized solutions do not depend on the macroscale deformation. Therefore, the homogenization has to be performed only once in each analysis. The homogenized stiffness tensors enter the constitutive relations, remaining constant until the end of the nonlinear damage analysis. When damage is initiated in the model, all stiffness tensors are being reduced by the term including the damage variable, as it can be seen from Eq. (8). In each finite element integration point, the incremental procedure is carried out, 
where the stress and double stress tensors are calculated from the updated strain tensor, second-order strain tensor and damage variable, as well as the stiffness tensors obtained in the preprocessing step. The presented damage formulation has been implemented into the twodimensional $C^{1}$ continuity triangular finite element [14] using the FE program ABAQUS and its user element subroutine UEL [19].

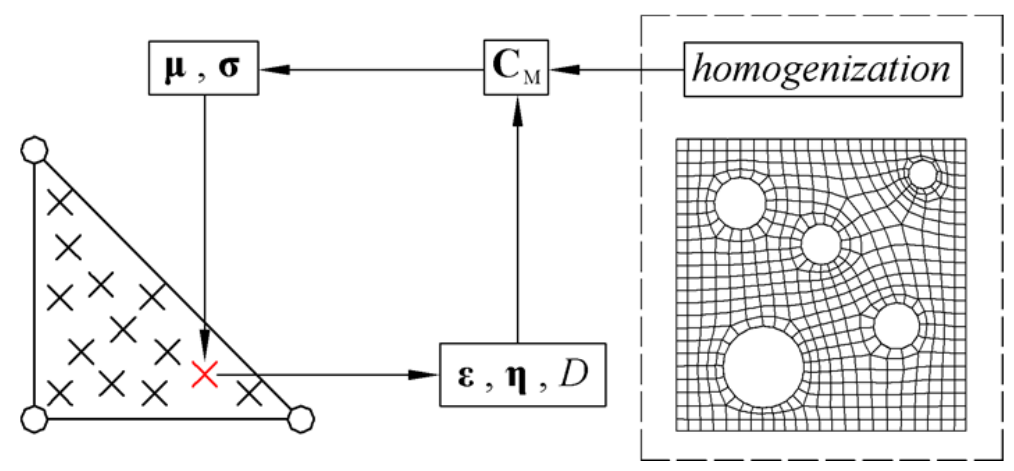

Figure 2: Scheme of the damage algorithm

\section{NUMERICAL EXAMPLE}

The algorithm presented above is verified on a benchmark problem already studied in [11] assuming homogeneous material. Here, the analysis is extended to heterogeneous materials.

As an example, a rectangular plate with an imperfect zone under tension is considered. The geometry and boundary conditions are shown in Fig. 3. The constitutive properties have been set to: Young's modulus $E=20000 \mathrm{~N} / \mathrm{mm}^{2}$, Poisson's ratio $v=0.25$, limit elastic strain $k_{0}=0.0001$, equivalent strain corresponding to the fully damaged state $k_{u}=0.0125$. The model is loaded by the horizontal displacement of $0.0325 \mathrm{~mm}$ at the right edge. In order to trigger localization, the Young's modulus is reduced by $10 \%$ in a $10 \mathrm{~mm}$ wide zone in the middle hatched area of the plate.

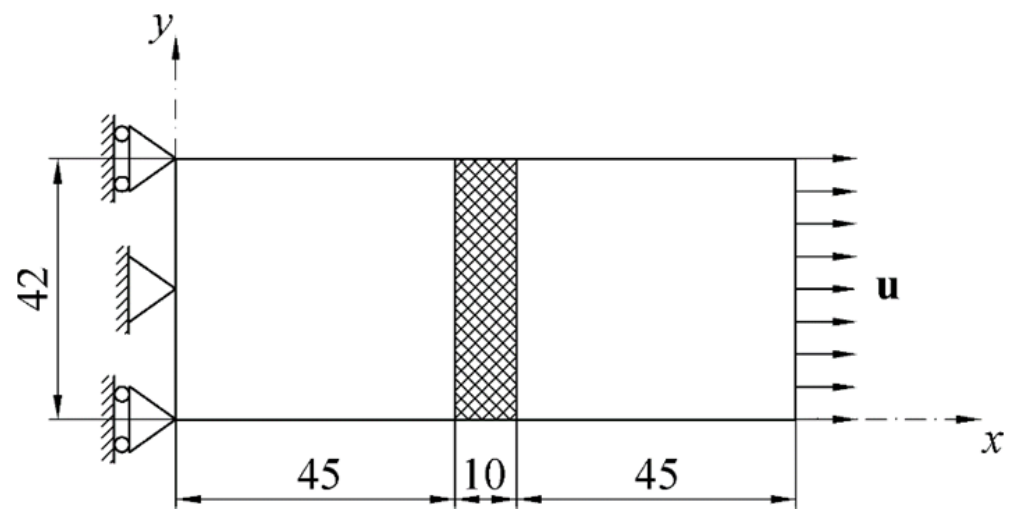

Figure 3: Geometry and boundary conditions of the plate model

The verification of the presented damage model is made using the results from [11], where the solutions are obtained in the same numerical example by means of the EFG meshless method. Therein, the constitutive tensors are derived for materials with granular microstructure, so the underlying microstructural theory differs when compared with the second-order homogenization approach. The constitutive model is restricted only to homogeneous materials, where the corresponding stiffness tensors are similar to those shown in Eq. (6). In [11] the stiffness tensors have been expressed in terms of the particle radius $r$ and they are used for the 
computation by means of the proposed algorithm. The damage responses obtained for the same microstructural values and using different approaches are presented in Fig. 4.

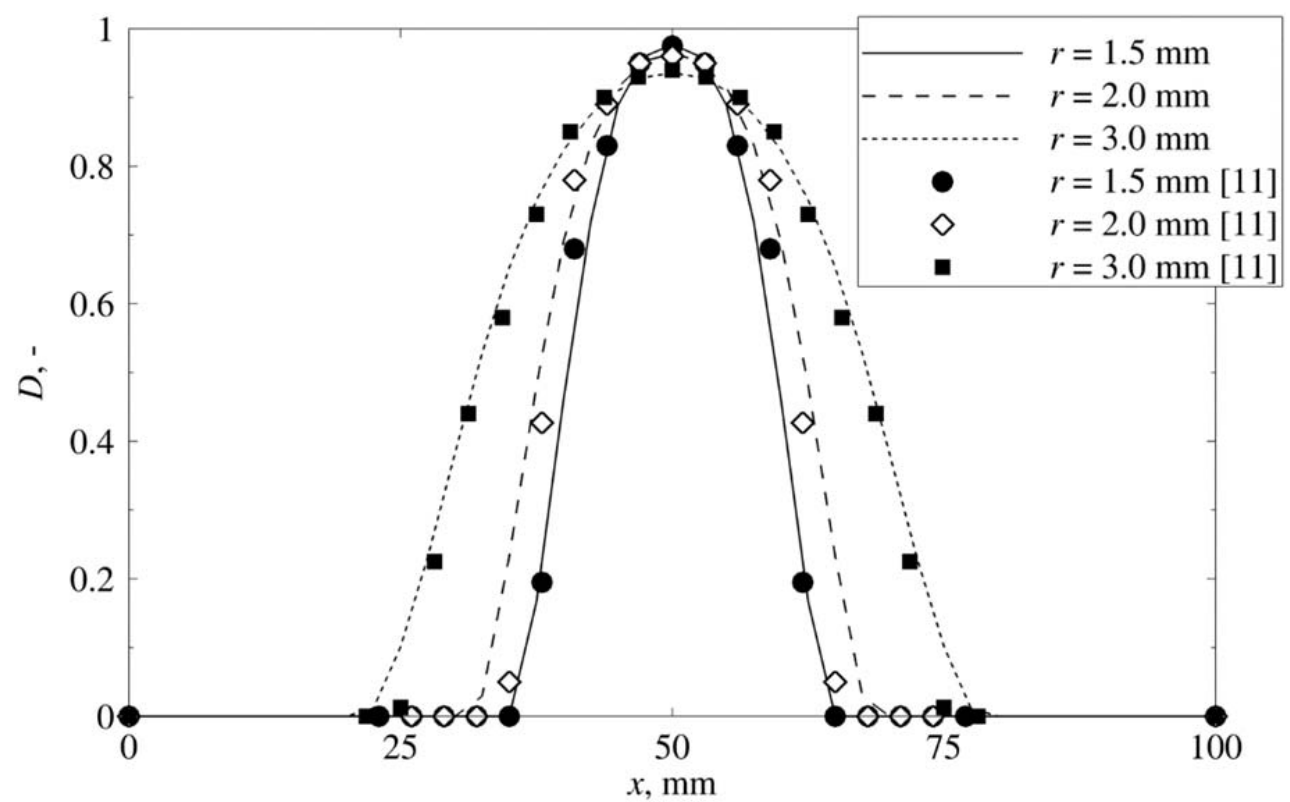

Figure 4: Comparison of damage profiles along the horizontal central axis obtained using the presented damage model with the results from the literature

As can be seen from Fig. 4, the calculated damage profiles show very good agreement with the solutions from the literature. The figure also illustrates the effect of the microstructural size on the behavior of the macroscale continuum. The increase in microstructural values leads to the expansion of the localization zone.

The evolution of the damage contour obtained with the microstructural parameter $l=1.5$ $\mathrm{mm}$ using the analytical expression of the constitutive tensors from [18], for the plate considered under imposed end displacement of $u=0.0325 \mathrm{~mm}$ is presented in Fig. 5. For the same microstructural parameter and the displacement, the contour plot of the equivalent elastic strain $\varepsilon_{e q v}$ is displayed in Fig. 6.

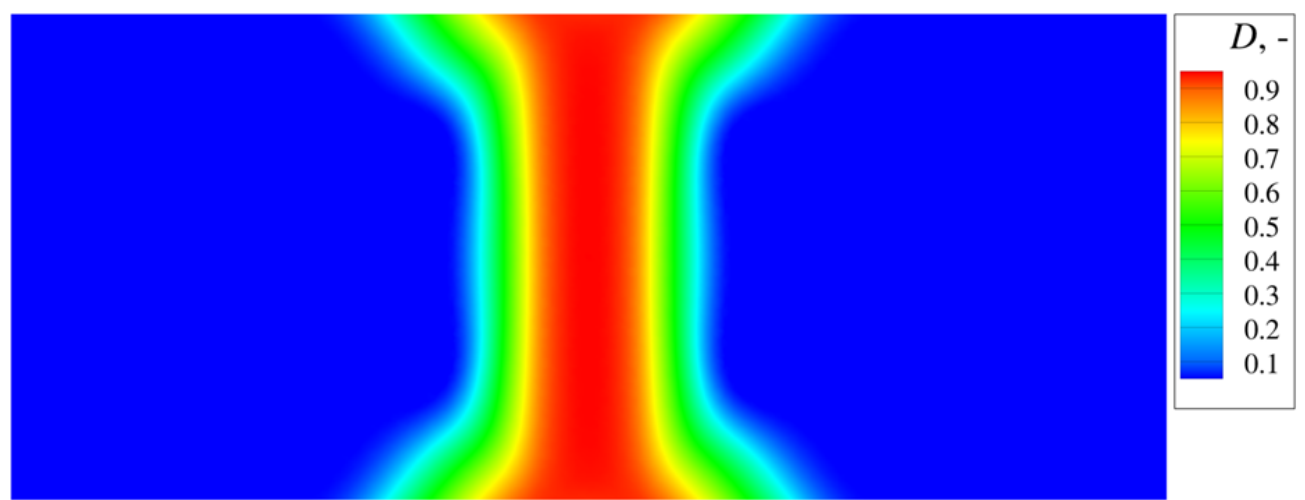

Figure 5: Distribution of damage $D$ for homogeneous material and internal length scale parameter $l=1.5 \mathrm{~mm}$ at $u=0.0325 \mathrm{~mm}$ 


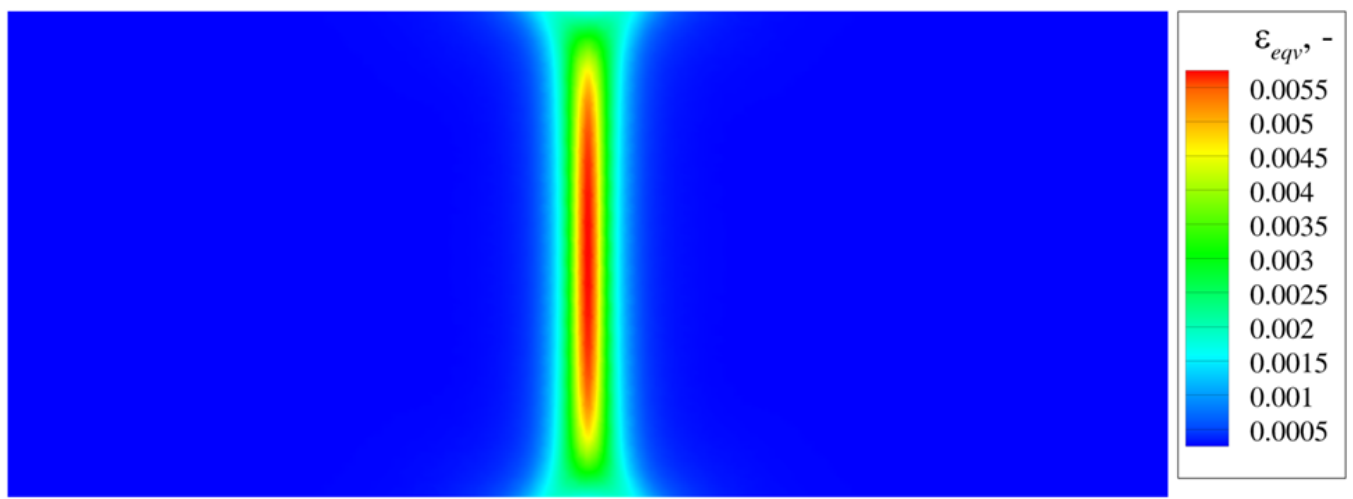

Figure 6: Distribution of equivalent elastic strain $\varepsilon_{\text {eqv }}$ for homogeneous material and internal length scale parameter $l=1.5 \mathrm{~mm}$ at $u=0.0325 \mathrm{~mm}$

In the analysis of the heterogeneous material, the full form of the incremental constitutive relations represented by Eq. (8) has to be solved. Therefore, the second-order homogenization has to be applied in the preprocessing step to obtain the material stiffness tensors. Here, two cases of simple academic RVE examples are analyzed and compared to the corresponding homogeneous solutions, i.e. the solutions emerging from the corresponding homogeneous materials of the same internal length scales. The two RVEs considered have the same average hole radius $r_{\text {ave }}$ and porosity $e$, but different size $L$, as shown in Fig. 7.

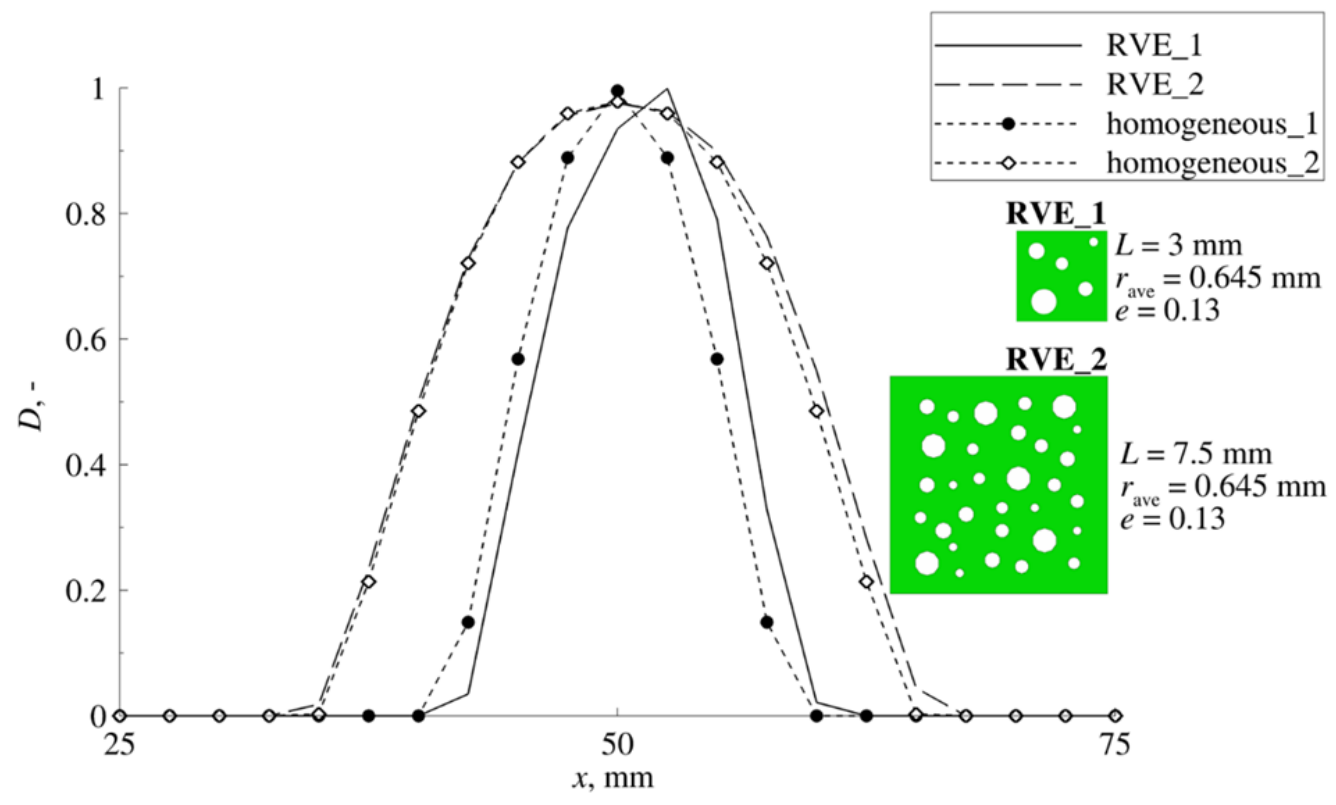

Figure 7: Comparison of damage profiles along the horizontal central axis obtained for two heterogeneous materials represented with RVEs of the same average hole radius $r_{\text {ave }}$ and porosity $e$, but different size $L$

In case of the smaller RVE, damage profile shows notable deviation from that obtained using the corresponding homogeneous material, while these differences are much smaller for the bigger RVE. This behavior can be explained with the positions of the holes as well as their distributions in the RVEs. These different damage responses can be confirmed by looking at the component values in the stiffness tensors $\mathbf{C}_{\sigma \eta}$ and $\mathbf{C}_{\mu \varepsilon}$, which are much bigger for the smaller RVE. It is to note that the influence of the RVE size and average hole radius on the 
damage response of heterogeneous structures is one of the open questions and should be investigated in forthcoming research.

\section{CONCLUSIONS}

A new computational approach employing the full strain gradient theory for the modeling of quasi-brittle softening phenomena has been proposed. This model is based on the isotropic damage law so that the material tangent stiffness matrices appearing in the constitutive relations are pre-multiplied by the same term governing the damage process. The highly nonlinear softening model is implemented into the triangular $C^{1}$ element using the FE software ABAQUS and provided UEL subroutines. The capabilities of the proposed computational strategy to simulate the strain localization has been demonstrated in a typical benchmark example consisting of a plate with an imperfect zone subjected to a tensile load. The verification of the algorithms derived has been made by the comparison with the solutions available in the literature, where the same problem has been analyzed using the EFG meshless method. Both homogeneous and heterogeneous material have been considered, where the latter is done by employing the second-order homogenization procedure to obtain the required material stiffness matrices. The homogeneous material has been analyzed employing the analytic expressions for the calculation of the stiffness matrices, and the softening state of the plate just prior to failure has been displayed by the contour plots of the damage variable and equivalent elastic strain field distributions. The heterogeneous material has been analyzed using the two different RVEs, where the damage responses have been compared to the homogeneous structures. It has been observed that the results depend on the RVE size and the number and position of the holes. The influence of heterogeneity on damage behavior requires more detailed studies which will be a topic of further research.

\section{ACKNOWLEDGMENT}

The work has been fully supported by Croatian Science Foundation under the project "Multiscale Numerical Modeling of Material Deformation Responses from Macro- to Nanolevel" (2516).

\section{REFERENCES}

[1] R.H.J. Peerlings, R. de Borst, W.A.M. Brekelmans, J.H.P. de Vree, Gradient enhanced damage for quasi-brittle materials, International Journal for Numerical Methods in Engineering, 39, 3391-3403, 1996.

[2] R. de Borst, L.J. Sluys, H.B. Mühlhaus, J. Pamin, Fundamental issues in finite element analysis of localization of deformation, Eng. Comput., 10, 99-121, 1993.

[3] C.S. Chang, L. Ma, Modeling of discrete granulates as micropolar continua, Journal of Engineering Mechanics, 116 (12), 2703-2721, 1990.

[4] L.J. Sluys, R. de Borst, Wave-propagation and localization in a rate dependent cracked medium model formulation and onedimensional examples, International Journal of Solids and Structures, 29, 2945-2958, 1992.

[5] R.H.J. Peerlings, Enhanced damage modeling for fracture and fatigue, Ph.D. Thesis. Eindhoven University of Technology, The Netherlands, 1999. 
[6] Z.P. Bažant, T. Belytschko, T.P. Chang, Continuum theory for strain-softening, J.Eng. Mech., 110, 1666-1692, 1984.

[7] R.H.J. Peerlings, R. de Borst, W. Brekelmans, M.G.D. Geers, Gradient-enhanced damage modeling of concrete fracture, Mechanics of Cohesive-Frictional Materials, 3 , $323-342,1998$.

[8] R. de Borst, H.B. Mühlhaus, Gradient-dependent plasticity: formulation and algorithmic aspects, Num. Int. J. Meth. Eng., 35, 521-539, 1992.

[9] L.J. Sluys, R. de Borst, Wave-propagation and localization in a rate dependent cracked medium model formulation and onedimensional examples, International Journal of Solids and Structures, 29, 2945-2958, 1992.

[10] C.S. Chang, H. Askes, L.J. Sluys, Higher-order strain/higher-order stress gradient models derived from a discrete microstructure, with application to fracture, Engineering Fracture Mechanics, 69, 1907-1924, 2002.

[11] Y. Yang, A. Misra, Higher-Order Stress-Strain Theory for Damage Modeling Implemented in an Element-free Galerkin Formulation, CMES: Computer Modeling in Engineering and Sciences, 64, 1-36, 2011.

[12] L. Kaczmarczyk, C.J. Pearce, N. Bićanić, Scale transition and enforcement of RVE boundary conditions in second-order computational homogenization, Int $J$ Numer Methods Eng, 74, 506-522, 2008.

[13] I.M. Gitman, H. Askes, L.J. Sluys, Representative volume: Existence and size determination, Engng. Fract. Mech., 74, 2518-2534, 2007.

[14] T. Lesičar, Z. Tonković, J. Sorić, A second-order two-scale homogenization procedure using C-1 macrolevel discretization, Computational Mechanics, 54 (2), 425-41, 2014.

[15] F. Putar, T. Lesičar, J. Sorić, Z. Tonković, Damage modeling using higher-order finite element formulation. I. Kožar, N. Bićanić, G. Jelenić, M. Čanađija eds. Proceedings of the $8^{\text {th }}$ International Congress of Croatian Society of Mechanics, CD edition. Opatija, Croatia, 2015.

[16] S. Akarapu, H.M. Zbib, Numerical analysis of plane cracks in strain-gradient elastic materials, International Journal of Fracture, 141 (3-4), 403-30.

[17] J. Lemaitre, A Course on Damage Mechanics, $2^{\text {nd }}$ edition. Springer, Berlin, 1996.

[18] V.G. Kouznetsova, M.G.D. Geers, W.A.M. Brekelmans, Size of a representative volume element in a second-order computational homogenization framework, International Journal for Multiscale Computational Engineering, 2 (4), 575-598, 2004.

[19] ABAQUS/Standard, in: Dassault Systemes, Providence, RI, USA, 2014. 